\title{
Does Complete Pathologic Response Come to Those Who Wait?
}

\author{
Nicole M. Geissen, DO ${ }^{1,2}$ \\ ${ }^{1}$ Division of Cardiothoracic Surgery, Department of Surgery, John H. Stroger, Jr. Hospital of Cook County, Chicago, IL; \\ ${ }^{2}$ Department of Cardiovascular Thoracic Surgery, Rush University Medical Center, Chicago, IL
}

Esophageal cancer remains a leading cause of cancer death worldwide, with multimodality therapy accepted as the standard of care. ${ }^{1}$ Neoadjuvant chemoradiotherapy prior to surgery has been shown to improve overall survival, with pathologic complete response (pCR) representing an independent predictor of overall survival. $^{2,3}$ While there are trials that suggest a neoadjuvant chemoradiation-to-surgery (CRT-S) interval of 6-8 weeks, there are no specific guidelines to support an optimum interval. ${ }^{4}$ Furthermore, the association of CRT-S with pCR has not been definitively established. To address this, Azab et al. retrospectively studied 5181 esophageal cancer patients [81\% adenocarcinoma, $18 \%$ squamous cell carcinoma (SCC)], using the National Cancer Data Base, who had CRT prior to definitive surgical resection, and examined several endpoints: overall survival, 90-day mortality, and pCR. The CRT-S interval was evaluated as a continuous variable, as well as a quintile system (Q1, 15-37 days; Q2, 38-45 days; Q3, 46-53 days; Q4, 54-64 days; Q5, 65-90 days), showing a significant increase of pCR rate and 90-day mortality across quintiles $(18 \%, 21 \%, 24 \%, 25 \%$, and $29 \%$, respectively, $p<0.001$; $5.7 \%, 6.2 \%, 6.8 \%, 8.5 \%$, and $8.2 \%$, respectively, $p=0.002)^{5}$

This was a well-done study with a large sample size of esophageal cancer patients with varying histologies $(81 \%$ adenocarcinoma, $18 \%$ SCC). The 3-year overall survival, with a mean of 33.9 months and median of 25.8 months, was in concordance with other studies for clinical stage III esophageal cancer. The rate of $\mathrm{pCR}$ for the study

(C) Society of Surgical Oncology 2019

First Received: 21 October 2018;

Published Online: 11 January 2019

N. M. Geissen, DO

e-mail: Nicole_Geissen@rush.edu population was $23 \%$ (1210/5181), which mirrors similar studies of smaller patient populations. ${ }^{2,3,5}$ Patients with $\mathrm{pCR}$, irrespective of histological type, were found to have comparative overall survival, regardless of CRT-S interval. While a longer CRT-S interval showed no negative impact in the SCC subpopulation, there was significantly worse OS and increased 90-day mortality across quintiles and with each additional week of CRT-S, especially over the last quintile (65-90 days). ${ }^{5}$

Limitations of this study include the lack of standardization for neoadjuvant treatment protocols, leaving ambiguity as to the dose of radiation administered, as well as concurrent versus sequential protocol. While the majority of patients in this study had distal adenocarcinoma, no information is provided regarding the breakdown of histologic type within the quintiles, except for the last quintile, 65-90 days. Analysis models were adjusted for histology as a predictor of mortality and OS, but not in the analysis of pCR to CRT-S. This is an important variable in that we know of the improved response of SCC of the esophagus compared with adenocarcinoma.

Azab and colleagues advocate for a predictive model of pCR to tailor timing of esophagectomy in patients with esophageal cancer. The findings of this study support a longer CRT-S interval to 65 days, after which OS and 90-day mortality suffer. While their data adds to previous studies supporting a longer CRT-S interval in association with $\mathrm{pCR}$, the determinants of $\mathrm{pCR}$ are still not completely understood. ${ }^{2,3,5}$ This study supports that the widely accepted window of a 6- to 8-week CRT-S interval is a positive predictor of $\mathrm{pCR}$. Until further studies can support a more pointed predictive model of $\mathrm{pCR}$, an interval of $<65$ days supports improved pCR and acceptable OS and 90-day mortality. 


\section{REFERENCES}

1. Franko J, Voynov G, Goldman CD. Esophagectomy timing after neoadjuvant therapy for distal esophageal adenocarcinoma. Ann Thorac Surg. 2016;101(3):1123-30.

2. Haisley KR, Laird AE, Nabavizadeh $\mathrm{N}$, et al. Association of intervals between neoadjuvant chemoradiation and surgical resection with pathologic complete response and survival in patients with esophageal cancer. JAMA Surg. 2016;151(11):e162734.

3. Shaikh T, Ruth K, Scott WJ, et al. Increased time from neoadjuvant chemoradiation to surgery is associated with higher pathologic complete response rates in esophageal cancer. Ann Thorac Surg. 2015;99(1):270-6.

4. Lin G, Han SY, Xu XP, Mao WM. Increasing the interval between neoadjuvant chemoradiotherapy and surgery in esophageal cancer: a meta-analysis of published studies. Dis Esophagus. 2016;29(8):1007-1114.

5. Azab B, Amudson J, Picado O, et al. Impact of chemoradiation-tosurgery interval on pathological complete response and short and long-term overall survival in esophageal cancer patients. Ann Surg Oncol. 2018. https://doi.org/10.1245/s10434-018-6897-4 (epub 11 Oct 2018). 